\title{
SIMULAÇÃO DAS COTAS DE EXTRAVASAMENTO PELO USO DA MODELAGEM HIDROLÓGICA E HIDRÁULICA EM UM TRECHO DO RIO MEIA PONTE - GO
}

\author{
José Alexandre Pinto Coelho Filho ${ }^{1}$, Matheus Fonseca Durães ${ }^{2}$, Maísa de Lourdes Martins \\ Araújo $^{3}$ e Cíntia de Lima Vilas Boas ${ }^{4}$ \\ 1,4 Pesquisador em Geociências, Serviço Geológico do Brasil - CPRM, e-mails: alexandre.coelho@cprm.gov.br ; \\ cintia.lima@cprm.gov.br \\ ${ }^{2}$ Professor, Departamento de Solos e Engenharia Agrícola, UFPR, e-mail: duraes@ufpr.br \\ ${ }^{3}$ Geógrafa, Consultora Ambiental, e-mail: maisaaraujo2007@gmail.com
}

\begin{abstract}
RESUMO: A avaliação do risco de inundação através da simulação hidráulica, por meio de vazões de projeto, permite identificar a necessidade de atividades de monitoramento, bem como otimizar os sistemas de alertas de inundações. Diante desse contexto, este trabalho teve por objetivo, analisar a possibilidade de extravasamento do rio Meia Ponte, localizado na cidade de Goiânia, Goiás, buscando contribuir para uma melhor ordenação do espaço urbano. Para isso, foram utilizados o modelo hidráulico HEC-RAS e dados da estação fluviométrica Montante de Goiânia, simulando tempos de retorno de 100, 500 e 1000 anos. As vazões para tempos de retorno de 100 anos $\left(182,9 \mathrm{~m}^{3} / \mathrm{s}\right), 500$ anos $\left(219,8 \mathrm{~m}^{3} / \mathrm{s}\right)$ e 1000 anos $\left(235,6 \mathrm{~m}^{3} / \mathrm{s}\right)$ implicam na elevação da linha de água para 3,53 metros, 3,78 metros e 4,38 metros na seção de régua $\left(S_{1}\right)$ e para 4,38 metros, 4,67 metros e 4,78 metros na seção normal $\left(S_{2}\right)$ da estação fluviométrica supracitada. Os resultados obtidos sugerem que as vazões simuladas não irão atingir cotas acima de 6,0 metros das réguas linimétricas nas seções da estação fluviométrica considerada, as quais controlam as vazões nesse trecho do rio Meia Ponte.
\end{abstract}

Palavras-chave: Inundação, tempo de retorno, vazões de projeto.

\section{SIMULATION OF EXTRAVASATION FLOW BY THE USE OF HYDRAULIC AND HYDROLOGIC MODELING IN A SECTION OF MEIA PONTE RIVER - GO}

\begin{abstract}
The flooding risk evaluation by hydraulic simulation through design flows allows to identify the need for monitoring activities and to optimize flood alert systems. In this context, this study aimed to analyze the possibility of leakage of the Meia Ponte River, located in Goiania city, Goiás State, seeking to contribute to a better ordering of urban space. To this end, were used the hydraulic model HEC-RAS and fluviometric records from the flow measuring station "Montante de Goiânia", simulating return periods of 100, 500 and 1000 years. The return period flows of 100 years $\left(182.9 \mathrm{~m}^{3} / \mathrm{s}\right), 500$ years $\left(219.8 \mathrm{~m}^{3} / \mathrm{s}\right)$ and 1000 years $(235.6 \mathrm{~m} / \mathrm{s})$ imply the rise of the waterline to $3.53 \mathrm{~m}, 3.78 \mathrm{~m}$ and $4.38 \mathrm{~m}$ in the rule section (S1) and to 4.38 meters, 4.67 meters and 4.78 meters in the normal section (S2) of the abovementioned station. The results suggest that the simulated flows will not reach the quotas
\end{abstract}


above $6.0 \mathrm{~m}$ levels on water rules at the sections of the considered fluviometric station, which control the flow that the Meia Ponte River stretch.

Keywords: Flooding, return period, design floods.

\section{INTRODUÇÃO}

O processo de ocupação e urbanização de áreas adjacentes a rios ocorre, na maioria das vezes, de forma irregular e desordenada, estando sujeitas ao risco de eventos de inundações, os quais são definidos por CPRM (2004) como fenômenos que ocorrem quando o volume de água que atinge simultaneamente o leito de um rio é superior à capacidade de drenagem de sua calha principal. Desta maneira, pode-se dizer que a inundação é caracterizada pelo preenchimento, em seção plena, da calha fluvial, onde qualquer acréscimo de vazão implica no transbordamento para a planície de inundação.

Nesse contexto, uma possibilidade em avaliar o risco de inundação é através da simulação de ocorrência de vazões de projeto, as quais servem como dados de entrada para simulação hidráulica da cota de inundação na seção transversal estudada, mediante modelagem hidráulica em softwares como o HEC-RAS - (Hydrologic Enginnering Center River Analisys System), desenvolvido pelo Corpo de Engenharia do Exército dos EUA (US Army Corps of Engineers, 2008).

O programa de simulação hidráulica HEC-RAS pode ser utilizado para a simulação do escoamento permanente e não permanente em canais naturais ou artificiais (USACE, 2010) e pode ser aplicado para a modelagem hidráulica em regime permanente ou em regime variável, como visto nos trabalhos de CPRM (2004), Ribeiro e Lima (2011), dentre outros.

As vazões de projeto podem ser calculadas para diferentes probabilidades de ocorrência, e suas respectivas cotas podem ser obtidas numa dada seção fluvial, contribuindo para identificar a necessidade do aumento de réguas linimétricas para monitoramento e controle de alertas de inundação, bem como na delimitação de zonas inundáveis em um plano diretor de ocupação de bacias hidrográficas. 
Segundo Fernandez et al. (2013), os modelos hidráulicos e hidrológicos espacialmente distribuídos utilizados para determinar áreas inundáveis, constituem uma importante ferramenta no planejamento e gestão de riscos, uma vez que possibilita a simulação de eventos extremos.

Dessa forma, o presente estudo tem por objetivo analisar a possibilidade de extravasamento do rio Meia Ponte, na seção da estação fluviométrica denominada "Montante de Goiânia”, no estado de Goiás, mediante a simulação de ocorrência de vazões de projeto com diferentes possibilidades de ocorrência.

\section{MATERIAIS E MÉTODOS}

\section{1 Área de Estudo}

A estação fluviométrica denominada como "Montante de Goiânia" (código 60640000), operada pelo Serviço Geológico do Brasil (CPRM), encontra-se aproximadamente à latitude $16^{\circ} 36^{\prime} 49^{\prime}$ ' Sul e longitude $49^{\circ} 16^{\prime} 47^{\prime}$ ' Oeste, na cidade de Goiânia, com área de drenagem de $1.740 \mathrm{~km}^{2}$, com registros fluviométricos datando desde o ano de 1975.

A cidade de Goiânia, capital do estado de Goiás, está inserida na bacia hidrográfica do rio Meia Ponte, a qual abrange 39 municípios e é a bacia mais densamente povoada do Estado de Goiás (Cunha, 2007).

A localização geográfica da área de estudo e da estação fluviométrica considerada é mostrada na Figura 1. 

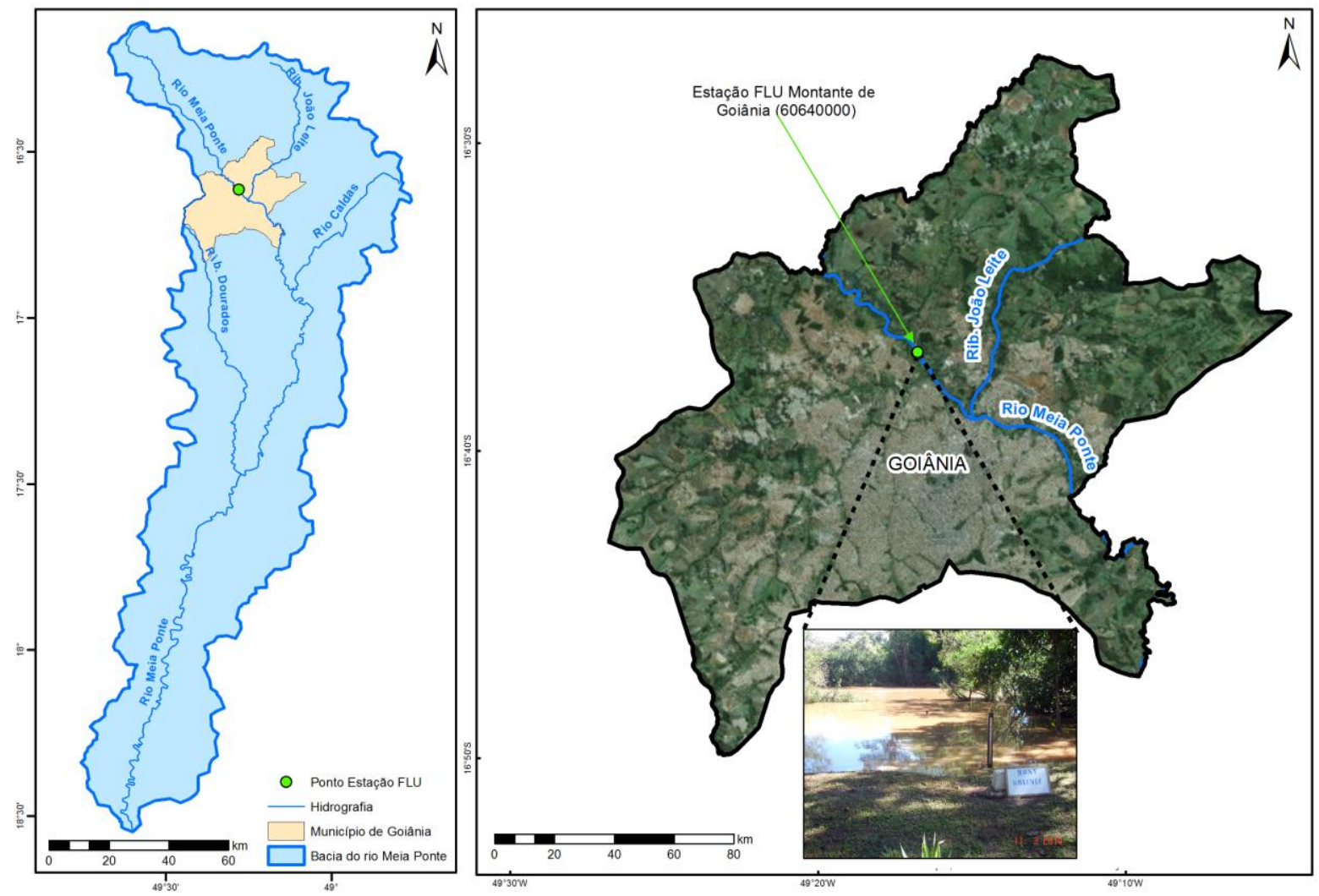

Figura 1 - Localização da área de estudo e da estação fluviométrica "Montante de Goiânia".

\subsection{Cálculo das vazões máximas}

Para o cálculo das vazões máximas foi aplicada a metodologia da análise de frequência, procedimento que tem como objetivo relacionar a magnitude dos eventos de cheia com sua frequência de ocorrência, utilizando uma função paramétrica de distribuição de probabilidades.

A análise de frequência de eventos extremos de vazões tem como objetivo permitir a estimativa de valores associados a uma probabilidade de excedência, ou seja, a probabilidade de ocorrer um evento de cheia com magnitude igual ou maior a um certo valor (Naghettini e Pinto, 2007). 
A verificação do ajuste da distribuição de probabilidades aos dados amostrais pode ser realizada pela aplicação dos testes do Qui-Quadrado e Komogorov-Smirnov, conforme apresentado em Costa Neto (1977) e Haan (1979).

Dessa forma, os valores das vazões máximas anuais registradas pela estação fluviométrica estudada foram ajustados a modelos de distribuições de probabilidades, para o cálculo das vazões máximas com os tempos de retorno de 100, 500 e 1000 anos.

Para a estimação dos parâmetros das distribuições de probabilidades candidatas, foram aplicados o método MOM e o método MVS. Foram ajustados os seguintes modelos distributivos: LogNormal 2p, Gumbel, Pearson-III, Generalizada de Valores Extremos (GEV) e LogPearson-III. O tempo de retorno $\mathrm{T}$ (anos) e a probabilidade de excedência, $\mathrm{P}(\mathrm{Q} \geq \mathrm{q})$, estão relacionados pela seguinte equação:

$P(Q \geq q)=\frac{1}{T(\text { anos })}$

A série histórica analisada compreende os registros dos dados de vazão entre os anos de 1975 e 2015. Esses registros resultam em uma série histórica de 37 dados de vazões máximas anuais, considerando seu ordenamento conforme o ano hidrológico.

De acordo com Yevjevich (1972), as falhas nas observações que compõem a série histórica podem resultar na possibilidade de grandes incertezas quanto às estimativas de parâmetros estatísticos. Dessa forma, não foram considerados na analise os dados dos anos que apresentaram meses com mais de 10 falhas de registro de observação de dados de vazão.

Para a estimativa dos parâmetros das distribuições de probabilidade, foram utilizados o método dos momentos (MOM) e o método da máxima verossimilhança (MVS). De acordo com Naghettini e Pinto (2007), o método MOM consiste em igualar os momentos populacionais aos momentos amostrais.

Assim, sejam $x_{1}, x_{2}, \ldots, x_{n}$, os valores observados constituintes de uma amostra aleatória simples retirada de uma população de variável aleatória distribuída conforme a função densidade $f_{X}=y ; \theta_{1}, \theta_{2}, \ldots, \theta_{k}$, de $k$ parâmetros, com momentos populacionais $\mu_{i}$. Se 
$m_{i}$ representam os momentos amostrais, o sistema de equações do método dos momentos baseia-se em:

$$
\mu_{i}=\theta_{1}, \theta_{2}, \ldots, \theta_{k}=m_{i} ; \quad i=1,2, \ldots, k
$$

As soluções $\hat{\theta}_{1}, \hat{\theta}_{2}, \ldots, \hat{\theta}_{k}$ do sistema formado pelas $k$ equações 2 , fornecem as estimativas dos parâmetros da distribuição.

De acordo com Bussab e Morettin (2004), o princípio da verossimilhança consiste em se maximizar uma função dos parâmetros da distribuição. $\mathrm{O}$ equacionamento para a condição de máximo resulta em um sistema de igual número de equações e incógnitas, para as quais as respectivas soluções produzem os estimadores de máxima verossimilhança.

Formalmente, seja uma amostra aleatória simples $x_{1}, x_{2}, \ldots, x_{n}$, extraída de uma população de uma variável aleatória distribuída conforme a densidade de probabilidades $f_{X}=y ; \theta_{1}, \theta_{2}, \ldots, \theta_{k}$. Sendo os dados amostrais considerados independentes, a distribuição de probabilidades conjunta desses elementos é dada por:

$$
L\left(\theta_{1}, \theta_{2}, \ldots, \theta_{k}\right)=\prod_{i=1}^{N} f_{X}\left(y ; \theta_{1}, \theta_{2}, \ldots, \theta_{k}\right)
$$

A equação 3 é conhecida como a função de verossimilhança, a qual maximizada consiste no método da máxima verossimilhança. A busca para se obter a condição de máximo da equação 3 implica no seguinte sistema de $k$ equações e $k$ incógnitas:

$$
\frac{\partial L\left(\theta_{1}, \theta_{2}, \ldots, \theta_{k}\right)}{\partial \theta_{i}}=0 ; \quad i=1,2, \ldots, k
$$

As soluções do sistema 4 , formado pelas $k$ equações, fornecem as estimativas dos parâmetros de distribuição.

Dessa maneira, estimados os parâmetros das distribuições de probabilidades, as mesmas podem ser ajustadas à série histórica de vazões máximas anuais para a estimativa das vazões máximas de tempo de retorno de 100, 500 e 1000 anos, correspondendo à probabilidade de ocorrência de $1 \%, 0,2 \%$ e $0,1 \%$, respectivamente. 


\subsection{Modelagem hidráulica}

A aplicação da metodologia da análise de frequência permite a obtenção do valor da vazão máxima para os tempos de retorno de 100 e 500 e 1000 anos, que serve como ponto de partida para a aplicação do programa HEC-RAS para a simulação da linha d'água na região da estação fluviométrica avaliada.

O software utiliza o Standard Step Method, que consiste em um método numérico para a estimativa do perfil da linha de água em canais abertos com regime permanente e escoamento gradualmente variado. Através da equação de energia, a seguir, pode-se verificar as mudanças no nível da linha de água entre as seções transversais.

$$
Y_{2}+Z_{2}+\frac{\alpha_{2} V_{2}^{2}}{2 g}=Y_{1}+Z_{1}+\frac{\alpha_{1} V_{1}^{2}}{2 g}+h_{e}
$$

Onde: $Y_{1}$ e $Y_{2}$ são profundidades das seções transversais; $Z_{1}$ e $Z_{2}$ são cotas do fundo do canal; $V_{1}$ e $V_{2}$ são velocidades médias; $\alpha_{1}$ e $\alpha_{2}$ são coeficientes de velocidade; $g$ é a aceleração da gravidade; e $h_{e}$ corresponde à perda de energia.

Para a modelagem utilizando o programa HEC-RAS, é necessário a definição do coeficiente de Manning, o qual está relacionado com os fatores de atrito do fluxo do escoamento na calha fluvial. Dessa maneira, o coeficiente de Manning relaciona-se com a tipologia do material que constitui a seção fluvial analisada. No presente estudo adotou-se o coeficiente de Manning correspondente a 0,35 (seções naturais), conforme recomendado por Baptista e Lara (2003).

Também é necessário o conhecimento da seção transversal do trecho fluvial em análise, o qual foi obtido por meio de levantamento de seção executado pela CPRM no mês de julho de 2014 e que reproduz a geometria da calha fluvial.

O levantamento de seção compreende a batimetria do curso de água na seção das réguas (no ponto das réguas linimétricas) e na seção normal (250 metros a montante da seção das réguas). A Figura 2 ilustra a área em estudo e as respectivas seções fluviais consideradas para a modelagem hidráulica. 


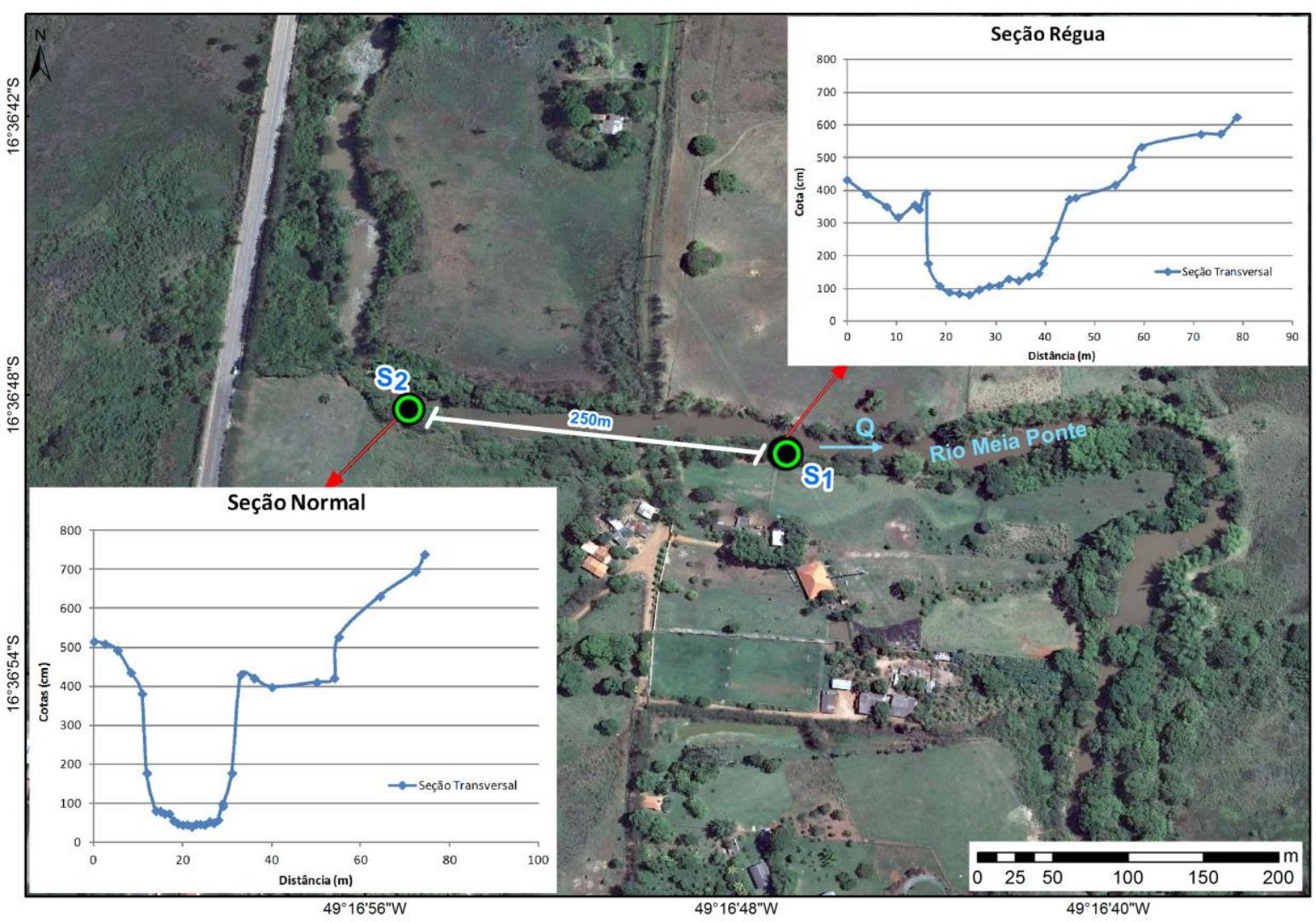

Figura 2 - Seções fluviais utilizadas na modelagem hidráulica.

Dessa maneira, foram simuladas as cotas nessas duas seções fluviais para os respectivos quantis de vazões máximas calculadas, associadas a tempos de retorno de 100, 500 e 1000 anos.

\section{RESULTADOS E DISCUSSÃO}

\subsection{Vazões Máximas}

Os quantis de vazões máximas para diversos tempos de retorno utilizando-se diferentes distribuições de probabilidades, obtidos a partir dos dados de vazão observados na estação fluviométrica "Montante de Goiânia”, são apresentados na Figura 3. 


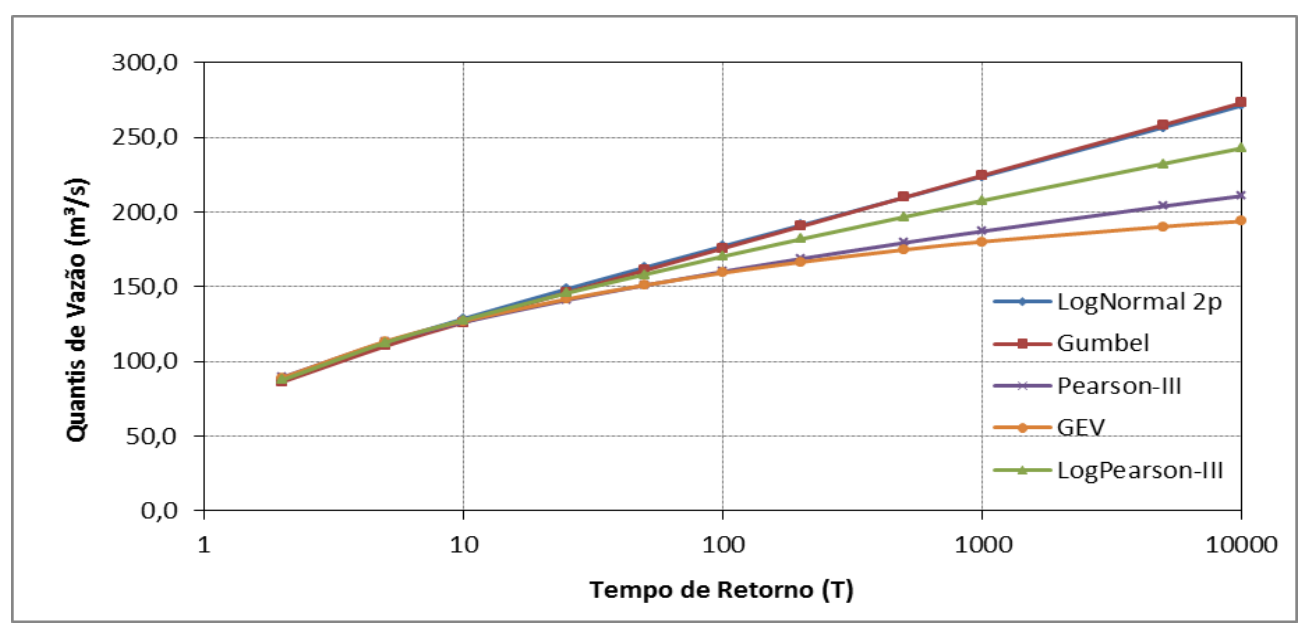

Figura 3 - Quantis de vazões máximas para diversos tempos de retorno utilizando-se diferentes distribuições de probabilidades, obtidos a partir dos dados de vazão observados na estação fluviométrica "Montante de Goiânia"

Os testes de aderência realizados (Qui-Quadrado e Kolmogorov-Smirnov), em nível de significância de 5\%, apontaram a aplicabilidade dos modelos distributivos utilizados.

Aplicando-se o método MVS, foi possível o ajuste apenas às distribuições de Gumbel e LogNormal 2p. Ou seja, apenas esses dois modelos distributivos apresentaram ajustes utilizando-se o método MOM e MVS para a estimação de seus parâmetros.

Os resultados dos quantis de vazão calculados para diferentes tempos de retorno, utilizando o modelo distributivo de Gumbel e LogNormal 2p, são apresentados na Tabela 1.

Tabela 1 - Comparativo dos quantis de vazão máxima, para diferentes tempos de retorno, utilizando-se os métodos MOM e MVS para a estimação dos parâmetros das distribuições de Gumbel e LogNormal 2p.

\begin{tabular}{ccccccccccccc}
\hline \multicolumn{10}{c}{ Comparativo Método dos Momentos x Método da Máxima Verossimilhança } \\
\hline \multirow{2}{*}{ Distribuição } & \multicolumn{10}{c}{ Tempo de Retorno } \\
\cline { 2 - 15 } & 2 & 5 & 10 & 25 & 50 & 100 & 200 & 500 & 1000 & 5000 & 10000 \\
\hline Gumbel (MOM) & 86,4 & 110,3 & 126,1 & 146,2 & 161,0 & 175,8 & 190,5 & 209,9 & 224,5 & 258,5 & 273,2 \\
Gumbel (MVS) & 86,3 & 112,1 & 129,3 & 150,9 & 167,0 & 182,9 & 198,8 & 219,8 & 235,6 & 272,4 & 288,2
\end{tabular}




$\begin{array}{cccccccccccc}\text { LogNormal (MOM) } & 86,8 & 112,4 & 128,6 & 148,5 & 162,9 & 177,1 & 191,2 & 209,8 & 223,8 & 256,9 & 271,4 \\ \text { LogNormal (MVS) } & 86,8 & 112,4 & 128,6 & 148,5 & 162,9 & 177,1 & 191,2 & 209,8 & 223,8 & 256,9 & 271,4\end{array}$

Em função de se encontrar diferentes valores para os quantis estimados, faz-se necessário o uso de testes de aderência para verificar se a distribuição usada pode ser aplicada ao conjunto de dados da variável que está sendo analisada, uma vez que esses testes procuram medir e avaliar os desvios entre a distribuição amostral e a teórica, entre eles, destacam-se os testes de KolmogorovSmirnov, Qui-quadrado e Anderson-Darling (Alves et al., 2013).

Contudo, para a modelagem hidráulica foram utilizados os quantis obtidos pela estimativa dos parâmetros da distribuição de Gumbel pelo método MVS, uma vez que esses valores foram superiores em relação àqueles obtidos pela aplicação do método MOM na estimativa dos parâmetros desse modelo distributivo, e também do método MOM e MVS na estimativa dos parâmetros da distribuição LogNormal $2 \mathrm{p}$. Por isso, representam a condição mais crítica em termos de produção de vazão no trecho em análise, e consequentemente de geração de maiores cotas de inundação na modelagem hidráulica realizada.

Assim, os valores de vazão adotados no estudo são de 182,9 m³/s, 219,8 m³/s e $235,6 \mathrm{~m}^{3} / \mathrm{s}$, correspondentes aos tempos de retorno de 100, 500 e 1000 anos, respectivamente.

A Tabela 2 apresenta os resultados da estatística dos testes para as duas distribuições selecionadas.

Tabela 2. Resultados das estatísticas dos testes de aderência aplicados às distribuições selecionadas.

\begin{tabular}{ccccccc}
\hline \multirow{2}{*}{ Distribuição } & \multicolumn{2}{c}{ P-valor } & \multicolumn{2}{c}{ Estatística de teste } & \multicolumn{2}{c}{ Hipótese } \\
\cline { 2 - 7 } & KS & $\chi^{2}$ & KS & $\chi^{2}$ & KS & $\chi^{2}$ \\
\hline Gumbel (MOM) & 0,7369 & 0,8733 & 0,11 & 4,1325 & Aceita & Aceita \\
Gumbel (MVS) & 0,7981 & 0,7689 & 0,1039 & 2,93 & Aceita & Aceita \\
LogNormal (MOM) & 0,8069 & 0,8227 & 0,1029 & 3,4601 & Aceita & Aceita \\
LogNormal (MVS) & 0,8633 & 0,7714 & 0,096 & 2,952 & Aceita & Aceita \\
\hline
\end{tabular}

KS: Kolmogorov-Smirnov; $\chi^{2}$ : qui-quadrado 


\subsection{Modelagem Hidráulica}

As Figuras 4, 5 e 6 apresentam as seções para as vazões de tempo de retorno de 100 $\operatorname{anos}\left(\mathrm{Q}=182,9 \mathrm{~m}^{3} / \mathrm{s}\right), 500 \operatorname{anos}\left(\mathrm{Q}=219,8 \mathrm{~m}^{3} / \mathrm{s}\right)$ e 1000 anos $\left(\mathrm{Q}=235,6 \mathrm{~m}^{3} / \mathrm{s}\right)$, juntamente com os níveis de água gerados, após as simulações empreendidas no HEC-RAS. O termo Bank Sta, representado por um ponto vermelho na legenda dessas figuras, delimita as cotas de topo da seção.

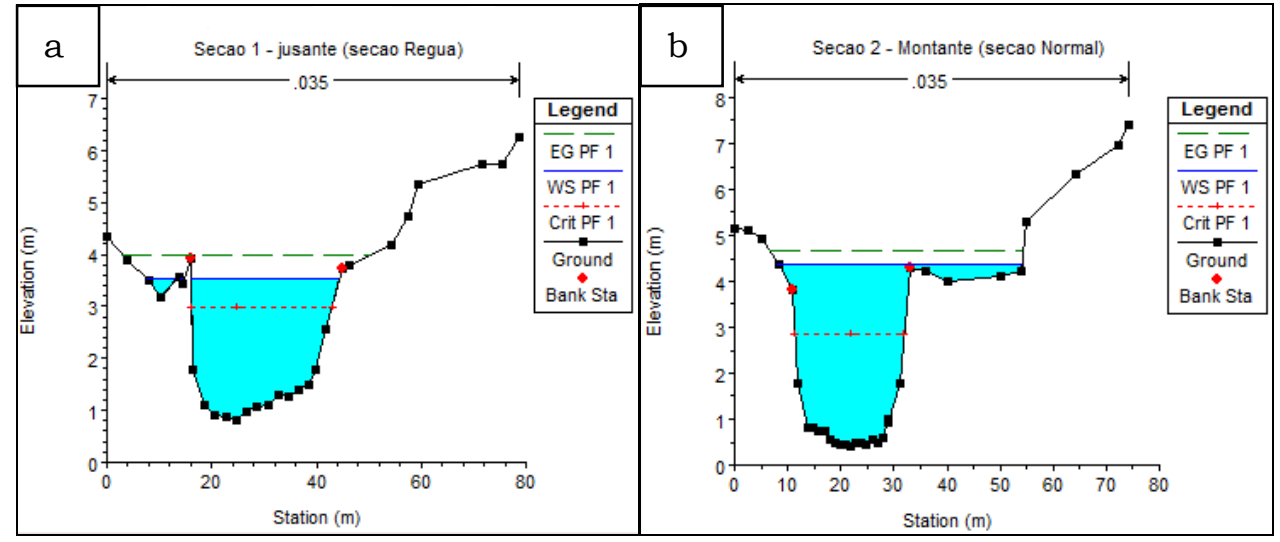

Figura 4 - Seções simuladas para a vazão de 100 anos de tempo de retorno $(\mathrm{Q}=182,9 \mathrm{~m}$ 3/s):

a) Seção 1 - Jusante (seção Régua), b) Seção 2 - Montante (seção Normal)

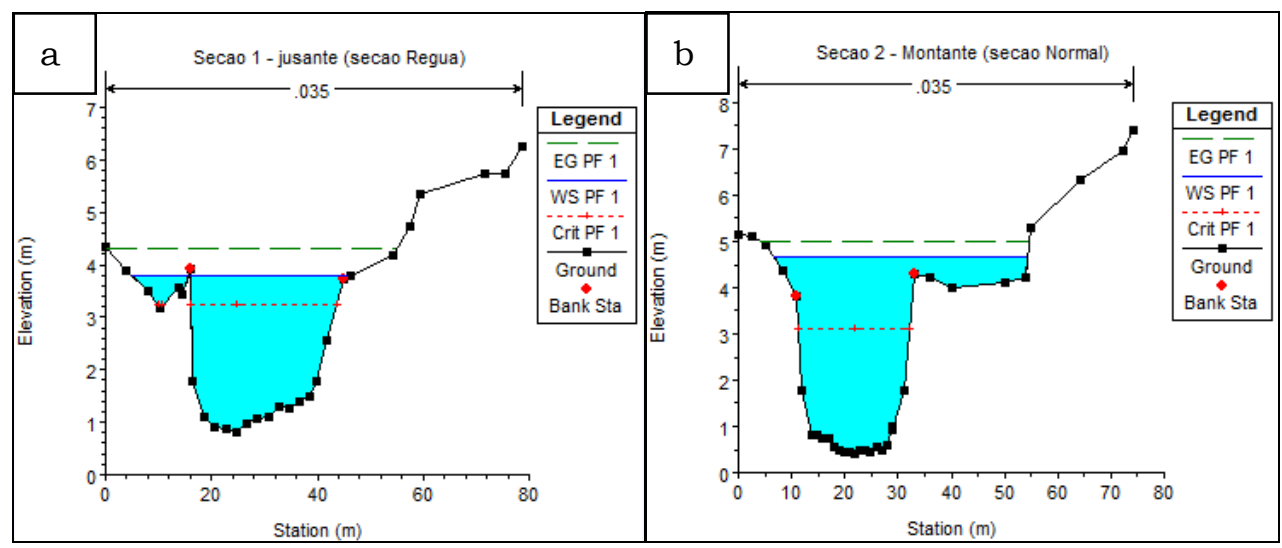

Figura 5 - Seções simuladas para a vazão de 500 anos de tempo de retorno $(\mathrm{Q}=219,8 \mathrm{~m}$ ³/s):

a) Seção 1 - Jusante (seção Régua), b) Seção 2 - Montante (seção Normal) 


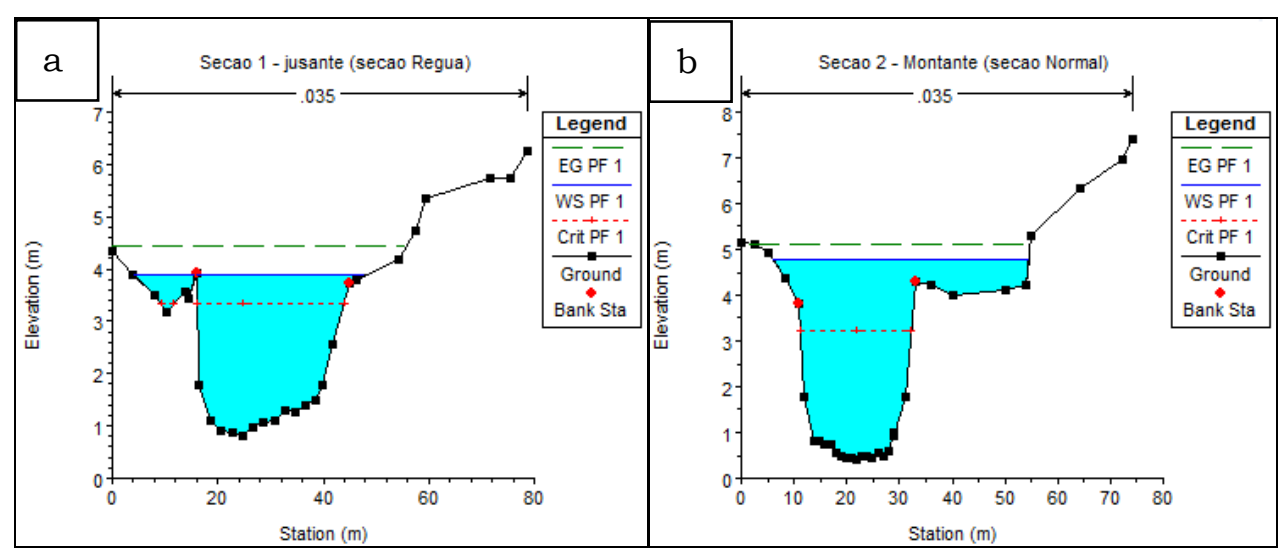

Figura 6 - Seções simuladas para a vazão de 1000 anos de tempo de retorno $(\mathrm{Q}=235,6$ m³/s): a) Seção 1 - Jusante (seção Régua), b) Seção 2 - Montante (seção Normal)

A Tabela 3 apresenta, pela ordem, os resultados numéricos da simulação hidráulica pela aplicação do programa HEC-RAS para o trecho fluvial em análise, considerando as vazões de projeto para os tempos de retorno de 100, 500 e 1000 anos, contemplando as cotas dos respectivos níveis de água para cada seção simulada.

Tabela 3 - Resultado da simulação no HEC-RAS para a vazão de 100, 500 e 1000 anos de tempo de retorno.

\begin{tabular}{ccccccccc}
\hline $\begin{array}{c}\text { Tempo de } \\
\text { retorno } \\
(\text { anos })\end{array}$ & Seção & Vazão & $\begin{array}{c}\text { Elevação } \\
\text { do fundo } \\
\text { do canal } \\
(\mathrm{m})\end{array}$ & $\begin{array}{c}\text { Elevação } \\
\text { da linha } \\
\text { d'água } \\
(\mathrm{m})\end{array}$ & $\begin{array}{c}\text { Velocidade } \\
(\mathrm{m} / \mathrm{s})\end{array}$ & $\begin{array}{c}\text { Área } \\
\text { molhada }\end{array}$ & $\begin{array}{c}\text { Largura } \\
\left(\mathrm{m}^{2}\right)\end{array}$ & Froude \\
\hline \multirow{2}{*}{100} & $\mathrm{~S}_{2}$ & 182,9 & 0,41 & 4,38 & 2,42 & 80,6 & 45,99 & 0,42 \\
& $\mathrm{~S}_{1}$ & 182,9 & 0,82 & 3,53 & 3,05 & 60,71 & 34,67 & 0,67 \\
\hline \multirow{2}{*}{500} & $\mathrm{~S}_{2}$ & 219,8 & 0,41 & 4,67 & 2,60 & 94,13 & 47,78 & 0,43 \\
& $\mathrm{~S}_{1}$ & 219,8 & 0,82 & 3,78 & 3,24 & 70,26 & 40,44 & 0,68 \\
\hline \multirow{2}{*}{1000} & $\mathrm{~S}_{2}$ & 235,6 & 0,41 & 4,78 & 2,66 & 99,44 & 48,46 & 0,44 \\
& $\mathrm{~S}_{1}$ & 235,6 & 0,82 & 3,88 & 3,32 & 74,22 & 43,69 & 0,68 \\
\hline
\end{tabular}

$\mathrm{Na}$ análise dos resultados apresentados, pode-se observar que para a vazão de tempo de retorno de 100 anos $\left(182,9\right.$ m³/s), a seção $S_{2}$ apresenta o nível de água na cota 4,38 m. Para as vazões de $500\left(219,8 \mathrm{~m}^{3} / \mathrm{s}\right)$ e $1000\left(235,6 \mathrm{~m}^{3} / \mathrm{s}\right)$ anos de tempo de retorno, o nível de água obtido foi de 4,67 m e 4,78 m, respectivamente. 
Na seção $S_{1}$, os níveis de água foram de $3,53 \mathrm{~m}, 3,78 \mathrm{~m}$ e 3,88 m para as vazões de $100(182,9 \mathrm{~m} / \mathrm{s}), 500\left(219,8 \mathrm{~m}^{3} / \mathrm{s}\right)$ e $1000\left(235,6 \mathrm{~m}^{3} / \mathrm{s}\right)$ anos de tempo de retorno, respectivamente.

Para as vazões simuladas nas seções fluviais consideradas, não foi verificado indicativo na mudança do regime de escoamento, estando o número de Froude (Fr) abaixo da unidade (escoamento subcrítico). Para a seção $S_{1}$, a modelagem indica um aumento de até 0,35 m na elevação do nível da água dependendo da vazão em transito na seção fluvial. Para a seção $S_{2}$ esse aumento chega a $0,40 \mathrm{~m}$.

Os resultados da modelagem indicam que, considerando a vazão de tempo de retorno de 1000 anos, pode-se considerar as elevações da linha d'água de 4,0 metros e 5,0 metros como as cotas de inundação para as seções $S_{1}$ e $S_{2}$, respectivamente.

\section{CONCLUSÕES}

Os modelos distributivos aqui considerados (Gumbel e LogNormal 2p) foram aceitos pela aplicação dos testes do Qui-Quadrado e Komogorov-Smirnov. Entretanto, optou-se pela utilização das vazões máximas obtidas pela aplicação da distribuição de Gumbel, com parâmetros obtidos pelo método MVS, uma vez que forneceu quantis superiores em relação ao método MOM e à aplicação da distribuição LogNormal 2p.

Dessa maneira, a modelagem hidráulica foi realizada para a condição de maior vazão estimada para o trecho em analise, com a geração de maiores cotas de inundação.

Os resultados obtidos sugerem que as vazões simuladas não irão atingir as cotas acima de 6,0 metros das réguas linimétricas nas seções da estação fluviométrica considerada, as quais controlam as vazões nesse trecho do rio Meia Ponte.

$\mathrm{O}$ estudo aqui apresentado pode ser futuramente ajustado e atualizado, uma vez que a base de dados de monitoramento de dados de vazão torna-se mais ampla e confiável ao longo do tempo, permitindo desdobramentos e melhorias futuras nos modelos aqui utilizados. 


\section{AGRADECIMENTOS}

Os autores agradecem à CPRM - Serviço Geológico do Brasil pelo apoio e pela disponibilização dos dados que viabilizaram a elaboração desse estudo.

\section{REFERÊNCIAS BIBLIOGRÁFICAS}

ALVES, A. V. P.; SANTOS, G. B. S.; MENEZES FILHO, F. C. M.; SANCHES, L. Análise dos métodos de estimativa para os parâmetros das distribuições de Gumbel e GEV em eventos de precipitações máximas na cidade de Cuiabá - MT. Revista Eletrônica de Engenharia Civil, v. 6, n. 1, p. 32-43, 2013.

BAPTISTA, M.B.; COELHO M.M.L.P. Fundamentos de Engenharia Hidráulica. 2.ed. Belo Horizonte: Editora UFMG, 2003. p. 241.

BUSSAB, W. O., MORETTIN, P. A. Estatística Básica. 5.ed. São Paulo: Editora Saraiva, 2004. 526p.

COSTA NETO, P. L. O. Estatística. Ed. Edgard Blücher LTDA, 1977. 264p.

CPRM - COMPANHIA DE PESQUISA DE RECURSOS MINERAIS. (Brasil) Definição da Planície de Inundação da Cidade de Governador Valadares - Relatório Técnico Final. Belo Horizonte, 2004.

CUNHA, M. A. C.; Evolução das Vazões do Rio Meia Ponte Jusante de Goiânia. In: XVII Simpósio Brasileiro de Recursos Hídricos, 2007, São Paulo-SP. Anais da ABRH. Porto Alegre-SP: ABRH, 2007. v. 1. p. 17.

FERNANDEZ, P. et al. Comparação dos modelos HEC-RAS e LISFLOOD-FP na delimitação de zonas inundáveis. Revista Recursos Hídricos, v. 34, p. 63-73, 2013. 
HAAN, C. T. Statistical methods in hydrology. 2. ed. Ames: The Iowa State University Press/Ames, 1979, 378p.

NAGHETTINI, M.; PINTO, E. J. A. Hidrologia Estatística. $1^{\text {a }}$ ed. Belo Horizonte: CPRM Companhia de Pesquisa de Recursos Minerais - Superintendência Regional de Belo Horizonte, 2007, 561p.

RIBEIRO, C. B. M.; LIMA, R. N. S. Modelagem Hidrológica e Hidráulica para Simulação de Inundações em uma Bacia Hidrográfica na Área Urbana do Município de Juiz de Fora/MG. In: XIX Simpósio Brasileiro de Recursos Hídricos, 2011, Maceió (AL). XIX Simpósio Brasileiro de Recursos Hídricos. Porto Alegre (RS): ABRH, 2011.

ROSTVEDT, J. O. et al. Summary of Floods in the United States during 1963, United States Government Printing Office, Washington, 1968, 130p.

U. S. Army Corps of Engineers - HEC-RAS. Hydrologic Modeling System - User's manual (version 2.1.2), Hydrologic Engineering Center, Davis. 2008.

U.S. ARMY CORPS OF ENGINEERS (2010). HEC-RAS River Analysis System: User's Manual, version 4.1. Hydrologic Engineering Center, 790 p.

YEVJEVICH, V. Flood and Society, in: Rossi, G.; Harmancioglu, N.; Yevjevich, V. (ed.) Coping With Floods, pp. 3 - 9, 1992. 